La Revue

des Droits

de l'Homme

\section{La Revue des droits de l'homme}

Revue du Centre de recherches et d'études sur les droits fondamentaux

Actualités Droits-Libertés | 2014

\title{
Précisions juridictionnelles sur l'octroi d'un droit de séjour aux ressortissants de pays tiers dans l'État membre d'origine du citoyen européen avec lequel ils nouent un lien familial
}

Droit de séjour et citoyenneté européenne (Directive 2004/38/CE et Art. 21 et 45 TFUE)

Maria Gkegka et Vincent Réveillère

\section{OpenEdition}

\section{Journals}

Édition électronique

URL : http://journals.openedition.org/revdh/639

DOI : $10.4000 /$ revdh.639

ISSN : 2264-119X

Éditeur

Centre de recherches et d'études sur les droits fondamentaux

Référence électronique

Maria Gkegka et Vincent Réveillère, « Précisions juridictionnelles sur l'octroi d'un droit de séjour aux ressortissants de pays tiers dans l'État membre d'origine du citoyen européen avec lequel ils nouent un lien familial », La Revue des droits de l'homme [En ligne], Actualités Droits-Libertés, mis en ligne le 01 avril 2014, consulté le 02 mai 2019. URL : http://journals.openedition.org/revdh/639 ; DOI : 10.4000/ revdh.639

Ce document a été généré automatiquement le 2 mai 2019.

Tous droits réservés 


\title{
Précisions juridictionnelles sur l'octroi d'un droit de séjour aux ressortissants de pays tiers dans l'État membre d'origine du citoyen européen avec lequel ils nouent un lien familial
}

Droit de séjour et citoyenneté européenne (Directive 2004/38/CE et Art. 21 et 45 TFUE)

\author{
Maria Gkegka et Vincent Réveillère
}

1 Saisie par voie préjudicielle par le Raad van State néerlandais, la Cour de justice de l'Union européenne a été invitée à déterminer si le droit de l'Union pouvait conférer un droit de séjour aux ressortissants de pays tiers, membres de la famille d'un citoyen européen, dans l'État de nationalité et de résidence dudit citoyen et, le cas échéant, à quelles conditions.

Dans les quatre cas, des ressortissants de pays tiers, O., B., S. et G., ont noué un lien familial avec un ressortissant néerlandais. Les personnes de référence ont, dans tous les cas, exercé leur liberté de circulation au sens du droit de l'Union. Toutefois, les quatre litiges recouvrent deux cas de figure assez différents et sont regroupés deux à deux par la juridiction néerlandaise. Le premier type de situation, celui de l'affaire O. et B. (C-456/12), concerne des cas de migration temporaire. Les citoyens de l'Union ont séjourné, en cette seule qualité, dans un autre État de l'Union avec les membres de leur famille ressortissants d'État tiers, avant de retourner dans l'État dont ils possèdent la nationalité. Le second type de situation, donnant lieu à l'affaire S. et G. (C-457/12), est celui des travailleurs frontaliers. Les citoyens de l'Union n'ont pas exercé leur droit de séjour dans un autre État membre et ils résident dans l'État membre dont ils ont la nationalité avec les membres de leur famille ressortissants d'État tiers, mais ils se rendent régulièrement dans un autre État membre dans le cadre de leurs activités professionnelles. Dans les deux affaires, la question qui se pose est la suivante : le droit de l'Union reconnait-il un droit de 
séjour aux membres de la famille de ces citoyens de l'Union, ressortissants d'États tiers, dans l'État de nationalité et de résidence de leur citoyen de référence?

Dans ces affaires, la reconnaissance de droits de séjour dérivé aux membres de leur famille ne poserait pas problème si elle n'intervenait pas précisément dans l'État de nationalité de celle-ci (v. les conclusions de l'avocat général $\mathrm{E}$. Sharpston qui estime que « les Pays-Bas refusent en substance d'octroyer un droit de séjour en vertu du droit de l'Union à des membres de la famille de leurs propres ressortissants qui sont ressortissants de pays tiers dans des cas où, en vertu du droit de l'Union, ils sont en principe tenus d'accorder de tels droits aux membres de la famille, ressortissants de pays tiers, de citoyens de l'Union qui ont la nationalité d'autres États membres. Il est curieux qu'un État membre souhaite ainsi traiter ses propres ressortissants de manière moins favorable que les autres citoyens de l'Union ", points 85 et 86). Ces arrêts interviennent ainsi dans le contexte du débat sur la question des «situations purement interne » et de la « discrimination à rebours ", discutées de manière extensive à propos de la jurisprudence récente de la Cour sur la privation de l'essentiel des droits du citoyen de l'Union (suivant CJUE, 8 mars 2011, Zambrano, Aff. C-34/09 - ADL du 11 mars 2011, Rec. p. I-01177, V. notamment les conclusions de Sharpston). Elles sont toutefois examinées dans ces arrêts à la lueur de solutions plus anciennes, développées dans la cadre du marché intérieur, les "personnes de référence » ayant exercé leur liberté de circulation.

Les positions prises in fine par la juridiction européenne conduisent en effet à l'adoption d'une solution cohérente au regard de sa jurisprudence passée. Dans la lignée des arrêts Singh et Eind, pour la première décision, et Carpenter pour la seconde, la Cour de justice étend les conséquences de ces solutions précédemment établies sur la situation des citoyens ainsi que des travailleurs européens. La nécessité de garantir l'exercice effectif des droits des citoyens et travailleurs européens aboutit donc, par ricochet, à l'octroi d'un droit au séjour aux ressortissants de pays tiers.

Pour fonder cette solution, la Cour s'appuie donc sur le lien de causalité entre la garantie d'un exercice effectif des droits de circulation du citoyen et du travailleur conférés par le TFUE, d'une part, et les droit de séjour des ressortissants d'un État tiers, membre de leur famille, d'autre part. Si elle s'attache d'abord à examiner la question au regard du droit dérivé, elle tâche ensuite d'explorer les pistes offertes par le droit primaire $\left(\mathbf{1}^{\circ}\right)$. Dans la première affaire, elle examine si l'article 21 TFUE peut constituer un fondement à l'octroi d'un droit de séjour $\left(2^{\circ}\right)$. Dans la seconde, elle vérifie si un droit de séjour peut être conféré en vertu de l'article 45 TFUE $\left(3^{\circ}\right)$. Tant le raisonnement mené, que la formulation employée, apparaissent remarquables et appellent dès lors un examen.

\section{$1 \%$ - Le refus de reconnaître un droit de séjour au regard du droit dérivé (directive 2004/38/CE)}

6 Dans les deux arrêts, la première question à laquelle la Cour a répondu est celle de savoir si les ressortissants d'un État tiers pouvaient bénéficier d'un droit de séjour dans l'État dont le membre de leur famille possède la nationalité en application de la directive 2004/38 du 29 avril 2004, relative au droit des citoyens de l'Union et des membres de leur famille de circuler et de séjourner librement sur le territoire des États membres.

7 La question de l'applicabilité de la directive est importante car elle prévoit un droit de séjour qui découle du seul statut de «membre de la famille» au sens de la directive, sans 
qu'aucune condition ne soit exigée (Iida, 57). Ces «membres de la famille » sont définis par la directive en son article 2, point 2 ) de façon très précise. Il s'agit du «conjoint », du " partenaire avec lequel le citoyen a contracté un partenariat enregistré» (sous certaines conditions de reconnaissance, V.b)), des « descendants directs qui sont âgés de moins de vingtet-un ans ou qui sont à charge, et les descendants directs du conjoint ou du partenaire » et des "descendants directs à charge et ceux du conjoint ou du partenaire ».

De manière non surprenante, la Cour va d'abord constater que les droits accordés par l'article 21, paragraphe 1, TFUE et les dispositions de la directive 2004/38 ne sont pas des droits autonomes mais dérivés (A), avant de décider que la directive ne confère aucun droit de séjour dérivé au membre de la famille d'un citoyen sur le territoire de l'État de nationalité de celui-ci (B).

\section{A - Le caractère dérivé des droits accordés aux membre de la famille du citoyen de l'Union}

La Cour rappelle tout d'abord que l'article 21, paragraphe 1, TFUE et les dispositions de la directive 2004/38 n'octroient "aucun droit autonome aux ressortissants d'États tiers 》. "En effet, ajoute-t-elle, les éventuels droits conférés à de tels ressortissants par les dispositions du droit de l'Union concernant la citoyenneté de l'Union sont des droits dérivés de l'exercice de la liberté de circulation par un citoyen de l'Union (voir, en ce sens, notamment les arrêts du 8 novembre 2012, Iida, C-40/11, non encore publié au Recueil, points 66 et 67, ainsi que du 8 mai 2013, Ymeraga et Ymeraga-Tafarshiku, C-87/12, non encore publié au Recueil, points 34 et 35) » (affaire O. et B., C-456/12, point 36, V. aussi affaire S. et G., C-457/12, point 33).

Le caractère dérivé des droits en cause ne saurait étonner. De manière incidente, on peut toutefois remarquer que celle-ci est quelque peu surprenante par sa généralité (même si, comme le montrent les références, ce point n'est pas nouveau à l'arrêt en question). En effet, la Cour exprime que les droits conférés à des ressortissants d'État tiers par «les dispositions du droit de l'Union concernant la citoyenneté de l'Union » sont dérivés de "l'exercice de la libre circulation par un citoyen de l'Union ». En effet, la formule semble difficilement conciliable avec des situations telles que celles en cause dans la jurisprudence Ruiz Zambrano. Si dans ces cas, les membres de la famille du citoyen de l'Union se voient bien reconnaître un droit dérivé, celui-ci est dérivé de la protection du statut de citoyen et non de l'exercice de sa liberté de circulation (v. CJUE, 8 mars 2011, Zambrano, Aff. C-34/09 ADL du 11 mars 2011).

\section{B - L'inapplicabilité de la directive 2004/38 aux membres de la famille du citoyen séjournant dans son État de nationalité}

11 Ensuite, la Cour décide qu'en vertu d'une analyse littérale, systématique et téléologique de ses dispositions, la directive 2004/38 ne confère pas de droit de séjour dérivé en faveur des ressortissants d'un État tiers, membres de la famille d'un citoyen de l'Union, dans l'État de nationalité de celui-ci (affaire O. et B., points 37s., auxquels renvoie l'affaire S. et G., point 34). En effet, la Cour interprète la directive comme ne visant que les situations dans lesquelles un citoyen de l'Union «se rend » ou « séjourne » dans un «État membre autre que celui dont il a la nationalité ». Ceci résulte pour le juge de la lettre de la directive (affaire O. et B., points 38 à 40) ainsi que de sa finalité (affaire O. et B., points 41 à 43). 

saurait être accordé que lorsque le citoyen a exercé son droit de libre circulation et s'établit dans un État membre autre que celui dont il a la nationalité. La Cour renvoie à différents arrêts dans laquelle elle exprimait cette solution concernant les droits conférés par l'art. 3, paragraphe 1 aux membres de la famille au sens de l'article 2, point 2) qui les accompagnent ou qui les rejoignent (v. CJCE, 25 juillet 2008, Metock e.a., C-127/08, Rec. p. I-6241, point 73; CJUE, Murat Dereci et autres c. Bundesministerium für Inneres, 15 novembre 2011, C-256/11, point 56 - ADL du 3 décembre 2011; CJUE, 8 novembre 2013, Lida, Aff. C-40/11, point 51; CJUE, 6 décembre 2012, O. e.a., C-356/11 et C-357/11, non encore publié au Recueil, point 41). En outre, elle souligne que l'interprétation d'autres dispositions de la directive confirment que le membre de la famille "ne peut pas se prévaloir, sur le fondement de cette directive, d'un droit de séjour dérivé dans l'État membre dont ce citoyen possède la nationalité » (v. CJUE, 5 mai 2011, McCarthy, aff. C-434/09, Rec. p. I-3375, point 37 - ADL du 10 mai 2011, ; CJUE, Lida, précité, point 64). Enfin, elle reprend le raisonnement qu'elle avait développé dans l'arrêt McCarthy, en vertu duquel, l'objet de la directive est l'exercice d'un droit de libre circulation et que comme le droit international "s'oppose à ce qu'un État membre refuse à ses propres ressortissants le droit d'accéder à son territoire et d'y séjourner à n'importe quel titre " (McCarthy, précité, point 29), elle «a uniquement vocation à régir les conditions d'entrée et de séjour d'un citoyen de l'Union dans les États membres autres que celui dot il a la nationalité » (affaire O. et B., point 42).

Ainsi, la Cour décide que la directive ne saurait conférer un droit de séjour aux membres de la famille d'un citoyen de l'Union dans l'état de nationalité de celui-ci. À cet égard, l'avocat général expliquait être "fermement convaincue " que la solution devrait être différente, mais elle considérait qu'une autre solution reviendrait à méconnaitre la directive (points 79s).

La directive n'étant pas applicable, la Cour de Luxembourg doit donc déterminer si les dispositions du TFUE peuvent fonder un droit de séjour dérivé dans les deux types de situation en cause.

\section{$2 \%$ L'octroi d'un droit de séjour en vertu de « l'effet utile » des droits de circulation du citoyen de l'UE (affaire 0 . et $B$ )}

La première affaire, O. et B. (C-456/12), concerne des membres de la famille de citoyens européens qui ont exercé leur droit de circulation en séjournant dans un État membre autre que celui dont ils sont ressortissants, avant de revenir dans l'État membre dont ils possèdent la nationalité. Le juge luxembourgeois doit déterminer en substance si l'article 21 paragraphe 1 TFUE peut fonder un droit de séjour dérivé pour ces ressortissants d'États tiers et, le cas échéant, à quelles conditions. Il répond en opérant deux transpositions. En premier lieu, il applique au domaine de la citoyenneté la notion d'« entrave à la sortie ", solution développée dans le cadre de la jurisprudence concernant le retour du travailleur dans son État d'origine. En second lieu, il applique au droit de séjour tiré du traité les conditions qui sont prévues pour le droit de séjour garanti par la 
directive. Le raisonnement développé repose notamment sur deux éléments, à savoir la jurisprudence européenne antérieure ainsi que sur le traité lui-même.

\section{A - «Effet utile » des droits de circulation du citoyen et droit de séjour des membres de sa famille}

La Cour examine donc si un droit de séjour dérivé peut être conféré à un ressortissant d'un État tiers dans l'État de nationalité du citoyen en vertu de l'article 21, paragraphe 1, TFUE, selon lequel «[t]out citoyen a le droit de circuler et de séjourner librement sur le territoire des États membres, sous réserve des limitations et conditions prévues par les traités et par les dispositions prises pour leur application». Elle est orientée en cela par la juridiction de renvoi qui remarque qu'un droit de séjour dérivé a été reconnu par la Cour aux membres de la famille du «travailleur » retournant dans son État d'origine et qui demande si, par analogie, un droit de séjour dérivé pourrait être reconnu aux membres de la famille du «citoyen» retournant dans l'État de nationalité de celui-ci (en vertu de l'article 21, paragraphe 2, TFUE) ou au " bénéficiaire d'une prestation de service » (en vertu de l'article 56 TFUE). Si la Cour a répondu par la positive à la première question, elle ne s'est néanmoins pas prononcée sur la seconde. Ce silence laisse penser que la réponse serait négative dans la mesure où elle a tendance à décider prioritairement en se fondant sur les libertés économiques (v. infra, l'affaire S. et G., l'avocat général, en revanche, examine ce cas de figure en l'intégrant dans un raisonnement plus général, $\mathrm{V}$. point 124s).

mener son raisonnement, la Cour établit un lien avec les arrêts Singh et Eind (arrêts du 7 juillet 1992, Singh, C-370/90, Rec. p. I-4265, et du 11 décembre 2007, Eind, C-291/05, Rec. p. I-10719). Elle conclut pour l'essentiel que bien que ces situations reposent sur le droit d'exercer des activités économiques, alors que les affaires $\mathrm{O}$. et $\mathrm{B}$. se situent, quant à elles, sur le terrain de la citoyenneté et du droit de séjour tel qu'il découle de l'article 21 TFUE, le même type d'entrave est essentiellement en cause.

Le raisonnement initialement développé dans le cas du retour du travailleur ayant exercé sa liberté de circulation repose principalement sur l'effet dissuasif qu'aurait le refus d'accorder un droit de séjour aux membres de sa famille. Un droit de "rentrer et de séjourner dans l'État membre dont il possède la nationalité " peut en effet être reconnu au travailleur migrant en vertu du Traité car «il est nécessaire pour assurer l'effet utile du droit de libre circulation que les travailleurs tirent de l'article 39 CE ainsi que des dispositions prises pour la mise en ceuvre dudit droit, telles que celles du règlement $n^{\circ} 1612 / 68$ " (Eind, point 32, aujourd'hui, art. 45 TFUE et directive 2004/38). En outre, la Cour explique que si un travailleur séjournant avec des membres de sa famille, d'origine non européenne, dans un autre État membre, n'était pas assuré que ceux-ci puissent revenir avec lui dans son État d'origine, il serait dissuadé d'exercer son droit de libre circulation. En effet, il ne pourrait bénéficier de la certitude de pouvoir continuer la vie familiale qu'il est susceptible de développer dans l'État d'accueil s'il souhaite revenir dans son État d'origine après son séjour dans un autre État membre (v. Eind, 35-36, v. aussi, Singh, points 19 et 20). Ainsi, le travailleur rentrant dans son État d'origine doit disposer « de facilités d'entrée et de séjour au moins équivalentes à celles dont il peut disposer, en vertu du traité ou du droit dérivé, sur le territoire d'un autre État membre » (Singh, point 19) et la directive concernant la liberté de circulation des travailleurs est appliquée par analogie (Singh, points 38s.).

Revenant sur cette jurisprudence dans ses conclusions sur 0 . et B., l'avocat général remarque que la rhétorique des deux arrêts est quelque peu différente, le second étant 
plus explicite sur la considération du droit « au regroupement familial » et souligne qu'il est rendu après l'insertion des dispositions relatives à la citoyenneté (point 94).

Transposé aux situations en cause, ce raisonnement implique qu'un citoyen de l'Union pourrait être dissuadé d'exercer ses droits conférés par le statut de citoyen, si la vie de famille qu'il avait développée ou consolidée à l'occasion de son séjour dans un Etat autre que celui dont il possède la nationalité n'était pas garantie après son retour dans l'Etat d'origine. Autrement dit, l'octroi d'un droit de séjour dérivé à un ressortissant d'un État tiers, membre de la famille de ce citoyen européen vise ici «à éliminer le même type d'entrave à la sortie de l'État membre dont il est originaire » (point 49).

Remarquons à cet égard que, contrairement à ce qu'elle avait fait dans l'arrêt Eind, la Cour se garde bien ici de mentionner le «statut de citoyen" ou sa "vocation fondamentale». Si ce dernier a pu être lu par certains comme symbolique d'un raisonnement plus orienté vers la protection des droits de la personne que vers celle de la liberté de circulation du travailleur, l'arrêt $\mathrm{O}$. B. tout en transposant la solution de «l'entrave à la sortie » au cas de la famille du citoyen migrant, exprime très clairement que sa justification réside dans l'effet « l'effet utile» des droits de circulation du citoyen de l'Union. "[L]a finalité et la justification » des droits de résidences dérivés qui peuvent être accordés est l'effet dissuasif de leur non reconnaissance sur la circulation du citoyen (point 45). C'est donc à cette lueur que la Cour détermine les types de séjours qui peuvent donner lieu à des droits de résidence et à quelles conditions.

\section{B - Caractère " effectif » du séjour et conditions de l'octroi des droits dérivés}

Pour qu'un droit de séjour dérivé puisse être accordé aux membres de la famille du citoyen de l'Union, certaines conditions portent sur le droit de séjour qu'ils ont exercé ensemble dans l'État hôte. En effet, la Cour considère d'abord que le séjour du citoyen dans l'État membre d'accueil doit être "caractérisé par une effectivité suffisante pour lui permettre de développer ou de consolider une vie de famille dans cet État membre» (point 51). Ensuite, elle précise que cette condition d'effectivité s'applique aussi bien au citoyen qu'au membre de sa famille et elle la lie étroitement aux droits et conditions de séjour prévus par la directives 2004/38.

23 Le caractère effectif semble devoir se déduire principalement de "l'installation » du citoyen dans cet État. En effet, la Cour utilise cette notion pour déterminer quelles catégories de droits de séjours prévues par la directive sont susceptibles de conduire à un séjour donnant droit à des droits dérivés. Ainsi, un séjour allant jusqu'à trois mois, prévu par l'article 6 paragraphe 1, ne satisfait pas aux conditions requises car ce séjour n'a pas pour fin "l'installation" du citoyen selon la Cour (point 52). L'effet cumulatif de différents séjours de courte durée dans l'État membre d'accueil, relevant de la même disposition, tels que des week-ends ou des vacances, n'est pas non plus en mesure d'ouvrir un droit de séjour dérivé (point 59). En revanche, un séjour de plus de trois mois et, a fortiori, l'acquisition d'un droit de séjour permanent en vertu, respectivement, de l'article 7, paragraphe 1, et de l'article 16, paragraphes 1 et 2, de la directive 2004/38 sont "de nature à aller de pair avec le développement ou la consolidation d'une vie de famille dans cet État membre» (point 53 à 55). 

l'Union et du membre de sa famille, ressortissant d'un État tiers, en vertu et dans le respect des conditions énoncées, respectivement, aux paragraphes 1 et 2 de l'article 7 ou de l'article 16 de la directive 2004/38 qui ouvre, au retour de ce citoyen de l'Union dans l'État membre dont il a la nationalité, un droit de séjour dérivé, sur le fondement de l'article 21, paragraphe 1, TFUE, au ressortissant d'un État tiers avec lequel ledit citoyen a mené une vie de famille dans l'État membre $»$ (point 56). le raisonnement. En effet, la Cour a décidé de les appliquer par analogie aux conditions d'octroi d'un droit de séjour dérivé dans l'État de nationalité du citoyen. Elle précise ainsi que «celles-ci ne devraient pas, en principe, être plus strictes que celles prévues par la directive 2004/38 pour l'octroi d'un tel droit de séjour à un ressortissant d'un État tiers, membre de la famille d'un citoyen de l'Union, qui a exercé son droit de libre circulation en s'établissant dans un État membre autre que celui dont il a la nationalité » (point 50). Partant, elle parvient à la conclusion que l'article 21 du traité exige, quant aux conditions de séjour du citoyen de l'Union dans un État membre autre que celui dont il possède la nationalité, que la directive 2004/38 «doit être appliquée par analogie » en dépit du fait qu'elle ne couvre pas un tel cas de retour. Cette solution découle, selon la Cour, du fait que, dans les deux cas, le citoyen européen est la «personne de référence» pour que le membre de sa famille puisse bénéficier d'un droit de séjour dérivé (point 50).

la de ces précisions apportées, la Cour entend également souligner que si le séjour doit être effectif, a fortiori, les dispositions du droit de l'Union ne devraient pas être utilisées à des fins abusives. La preuve de pratiques abusives implique de réunir un ensemble d'éléments objectifs "d'où il résulte que, malgré un respect formel des conditions prévues par la réglementation de l'Union, l'objectif poursuivi par cette réglementation n'a pas été atteint » et un élément subjectif " un élément subjectif consistant en la volonté d'obtenir un avantage résultant de la réglementation de l'Union en créant artificiellement les conditions requises pour son obtention" (point 58 ).

27 La Cour précise sa position en portant une appréciation sur le premier litige. Pour « 0 », titulaire d'une carte de séjour au titre de l'article 10 de la directive 2004/38 en tant que membre de la famille d'un citoyen de l'Union, la Cour relève que cette seule carte de séjour, ayant un caractère déclaratif et non pas constitutif de droits, n'est pas en mesure d'ouvrir un droit au séjour en vertu du droit de l'Union (point 60 ; v. en ce sens, Eind, précité, points 20 à 26 et arrêt du 21 juillet 2011, Dias, C-325/09, Rec. p. I-6387, points 42 à 49). Comme le souligne l'avocat général, ce qui compte est le traitement auquel le citoyen «avait droit» et non celui dont il a "effectivement bénéficié » (point 95). En revanche, la différence d'appréciation quant à la définition du séjour du citoyen pouvant donner lieu à la reconnaissance de droit aux membres de sa famille semble conduire à une solution différente pour une personne dans le cas de $\mathrm{O}$. Si l'appréciation de la situation est du ressort de la juridiction de renvoi, les directives que la Cour donne incitent à penser qu'une situation comme celle de 0 . ne pourrait donner lieu à un droit de séjour dérivé pour la Cour (point 59 et 60), l'avocat général concluant dans l'autre sens (point 143).

En effet, l'avocat général avait proposé une voie assez différente quant à la définition du séjour, en mettant l'accent sur le «lieu de résidence» du citoyen entendue comme «le centre habituel des ses intérêts " (v. les conclusions de l'avocat général E. Sharpston, points $96 \mathrm{~s})$. Or, au-delà du caractère dissuasif du refus de droit de séjour lors de la sortie initiale du citoyen, Mme Sharpston accorde une place importante à la liberté de celui-ci

La Revue des droits de l'homme , Actualités Droits-Libertés | 2014 
dans le choix de son lieu de résidence (v. nt, points 88 et 89 , point 95 et l'idée que les droits tirés du droit de l'Union font partie du «passeport » du citoyen de l'Union qu'il doit conserver à son retour dans l'État de nationalité). La « résidence » ne requiert pas une présence physique constante mais prépondérante (point 102), et est conciliable avec le maintien d'une forme de résidence dans l'État d'origine (point 103). Si la durée du séjour est alors un critère pertinent, il ne saurait y avoir de « seuil absolu » (point 111, v. aussi point 138).

La Cour de justice précise également sa position en portant une appréciation sur le second litige. Dans le cas de «B ", celui-ci ayant acquis la qualité de membre de la famille d'un citoyen de l'Union à un moment postérieur au séjour de sa partenaire dans l'État membre d'accueil et n'ayant pas pu bénéficier dans cet État d'un droit de séjour dérivé en vertu de la directive 2004/38 ne saurait invoquer l'article 21 TFUE. La Cour souligne ici bien le fait que le séjour "en vertu et dans le respect des conditions" énoncées par les dispositions de la directive concernant les séjours de plus de trois mois, est une condition pour l'établissement d'un éventuel droit de séjour dérivé.

En définitive, la Cour transpose dans cet arrêt aux citoyens migrants la notion d' «entrave à la sortie» en la fondant clairement sur «l'effet utile» de l'article 21, paragraphe 1, du TFUE. Cette solution a pour conséquence de limiter le champ des "situations purement internes " en reconnaissant une nouvelle situation dans laquelle un citoyen peut invoquer le droit de l'Union contre son État de nationalité. Elle permet de protéger la vie familiale de citoyens non actifs économiquement qui retournent dans leur État d'origine et conforte en ce sens le statut de citoyen de l'Union. La Cour donne toutefois des garanties aux États membres en définissant de manière claire les migrations concernées grâce à la notion de « séjour effectif » et à l'exigence d'un séjour en vertu et dans le respect des dispositions de la directive 2004/38 qui concernent les séjours supérieurs à trois mois. La possibilité d'invoquer le droit de l'Union contre son État d'origine fait donc suite à une «installation » à l'étranger de la part des citoyens et de leur famille.

\section{$3 \%$ - L'octroi d'un droit de séjour en vertu de l'effet utile du droit de circulation du travailleur (affaire S. et G.)}

31 La seconde affaire, S. et G. (C-457/12), concerne des membres de la famille de citoyens européens résident avec ceux-ci dans le pays de nationalité du citoyen. Dans les deux cas, le citoyen de l'Union se rend régulièrement dans un autre État membre pour travailler. Dans le litige concernant Mme G., le citoyen de l'Union travaille pour une société établie dans un État membre autre que celui où il réside. Dans l'affaire concernant Mme S., le citoyen de l'Union se rend régulièrement dans un État membre autre que celui de sa résidence dans le cadre de ses activités professionnelles mais la société qui l'a engagé est établie dans l'État membre de sa résidence.

Comme dans l'affaire précédente, la directive 2004/38 ne s'applique pas au séjour du citoyen et des membres de sa famille dans l'État de nationalité du citoyen. Les travailleurs en cause, qui ont fait usage de leur liberté de circulation, n'ont en revanche pas séjourné 
en dehors de leur État d'origine. La Cour doit donc déterminer si les articles 20 et 21 paragraphe 1TFUE, consacrés à la citoyenneté européenne, d'une part, et l'article 45 TFUE, consacré à la liberté de circulation des travailleurs, d'autre part, peuvent fonder un droit de séjour dérivé pour ces ressortissants d'États tiers et, le cas échéant, à quelles conditions. Elle ne traitera que de la seconde question. En effet, ayant répondu sur le terrain de la liberté de circulation du travailleur, elle estime qu'elle n'a pas à procéder à l'examen de la question sur celui de la citoyenneté car les articles 20 et 21 paragraphe 1 TFUE qui «énoncent de manière générale le droit pour tout citoyen de l'Union de circuler et de séjourner librement sur le territoire des États membres, trouvent une expression spécifique à l'article 45 TFUE en ce qui concerne la libre circulation des travailleurs » (point 45, V. not., arrêt C-100/01, Olazabal, Rec. p. I-10981, point 26 ou, cité par la Cour, arrêt du 4 juillet 2013, Gardella, C-233/12, non encore publié au Recueil, point 38).

La question centrale de l'arrêt était donc celle de savoir si les ressortissants de pays tiers se trouvant dans les situations évoquées pouvaient se prévaloir d'un droit de séjour en vertu de l'article 45 TFUE. La juridiction néerlandaise demandait également à la Cour si un raisonnement développé dans un autre cadre, celui de la libre prestation des services dans l'affaire Carpenter (arrêt du 11 juillet 2002, Carpenter, C-60/00, Rec. p. I-6279), pouvait être étendu aux situations en cause, concernant la libre circulation de travailleurs.

\section{A - « Effet utile » des droits de circulation du travailleur et droit de séjour des membres de sa famille}

En fondant son raisonnement sur sa jurisprudence antérieure (arrêt du 11 juillet 2002, Carpenter, C-60/00, Rec. p. I-6279) et sur l'article 45 TFUE, la juridiction européenne juge que l'octroi d'un droit de séjour à un ressortissant d'un Etat tiers membre de la famille d'un citoyen européen peut être nécessaire afin de garantir à ce dernier l'exercice effectif de la liberté fondamentale garantie par ladite disposition.

Dans le célèbre arrêt Carpenter (arrêt du 11 juillet 2002, Carpenter, C-60/00, Rec. p. I-6279), la Cour avait posé, comme elle le rappelle ici, que « l'article 56 TFUE, lu à la lumière du droit fondamental au respect de la vie familiale, s'oppose à ce que, dans une situation telle que celle dans l'affaire ayant donné lieu audit arrêt, l'État membre d'origine du prestataire de services établi dans ce même État, qui fournit des services à des destinataires établis dans d'autres États membres, refuse le droit de séjour sur son territoire au conjoint de ce prestataire, ressortissant d'un État tiers »(point 37).

Il convient de rappeler brièvement la situation en cause. L'épouse philippine d'un ressortissant britannique exerçant son droit à la libre prestation de service (depuis son État d'origine au profit de personnes établies sur le territoire d'États tiers et en se déplaçant dans ces derniers) s'était vu refuser une autorisation de séjour par le RoyaumeUni où elle résidait avec son mari et les enfants de ce dernier. Il est important de préciser qu'il n'était pas contesté qu'elle "jouait un rôle important dans l'éducation de ses beauxenfants » et "qu'elle pouvait être indirectement responsable du succès croissant de l'entreprise de son mari » (Carpenter, C-60/00, point 18). Au regard de ces circonstances, la Cour décide que «la séparation des époux Carpenter nuirait à leur vie familiale et, partant, aux conditions de l'exercice d'une liberté fondamentale par M. Carpenter. En effet, cette liberté ne pourrait pas produire son plein effet si M. Carpenter était détourné de l'exercer par les obstacles mis, dans son pays d'origine, à l'entrée et au séjour de son conjoint (voir, en ce sens, arrêt Singh, précité, point 23) 
» (Carpenter, C-60/00, point 39). En outre, elle précise qu'un État membre ne saurait justifier une entrave par des mesures qui seraient contraires aux droits fondamentaux dont la Cour assure le respect (point 40) et que la décision d'expulsion de Mme Carpenter "ne respecte pas un juste équilibre entre les intérêts en présence, à savoir, d'une part, le droit de M. Carpenter au respect de sa vie familiale et, d'autre part, la défense de l'ordre public et de la sécurité publique » (point 43).

Si cette solution est transposée aux litiges en cause, donc au cas du travailleur, la Cour ne le fait qu'avec une certaine circonspection. Pour le juge européen, «certes, l'interprétation de l'article 56 TFUE à laquelle la Cour a procédé dans l'arrêt Carpenter, précité, est transposable à l'article 45 TFUE. L'effet utile du droit de libre circulation des travailleurs peut en effet requérir qu'un droit de séjour dérivé soit octroyé à un ressortissant d'un État tiers, membre de la famille du travailleur, citoyen de l'Union, dans l'État membre dont ce dernier possède la nationalité » (point 40). Il ajoute ensuite que « toutefois, la finalité et la justification d'un tel droit de séjour dérivé se fonde sur la constatation que le refus de sa reconnaissance est de nature à porter atteinte à l'exercice des libertés fondamentales garanties par le traité FUE (voir, en ce sens, arrêts du 8 novembre 2012, Iida, C-40/11, non encore publié au Recueil, point 68; Ymeraga et YmeragaTafarshiku, précité, point 35, ainsi que du 10 octobre 2013, Alokpa e.a., C-86/12, non encore publié au Recueil, point 22). » (point 41).

La lecture de ces paragraphes laisse penser que les termes en ont été choisis avec précaution. "Certes", la Cour décide de transposer la jurisprudence, mais c'est en s'appuyant très clairement sur le fait qu'elle ne saurait être fondée autrement que par «l'effet utile» du droit de circulation des travailleurs. Les éventuels droits de séjour dérivés ne sont accordés que dans la mesure où leur refus mettrait en cause l'effectivité de la liberté de circulation du travailleur. La rhétorique, si ce n'est la solution, est donc assez différente de l'arrêt Carpenter. Il convient alors de relever que l'effet utile du droit de libre circulation des travailleurs exige qu'un droit de séjour dérivé soit octroyé à un ressortissant d'un État tiers, membre de la famille du travailleur européen dans l'État membre dont ce dernier possède la nationalité. À ce constat s'ajoute la condition classique liée à l'exercice effectif des droits consacrés par le traité. Or, le traité confère un tel droit de séjour dans la mesure où le refus de sa reconnaissance est susceptible de porter atteinte à l'exercice des libertés fondamentales garanties par le traité FUE.

\section{$B$ - Caractère « nécessaire » du séjour du membre de la famille}

39 Cette solution dégagée, la Cour poursuit en fournissant des éléments d'appréciation qu'il appartient à la juridiction de renvoi de prendre en compte lors de l'examen de la situation. Elle estime que celle-ci doit établir le caractère « nécessaire » du droit de séjour dérivé « afin de garantir » la liberté de circulation du travailleur (point 42). Revenant sur les circonstances de Carpenter et mentionnant, sans s'y référer, une situation qui semble correspondre à celle de $\mathrm{S}$, elle souligne que «le seul fait qu'il pourrait paraitre souhaitable qu'un tel accueil soit pris en charge par les ressortissant d'un État tiers, ascendant direct du conjoint du citoyen de l'Union, ne suffit pas à constater un tel caractère dissuasif » (point 43). La Cour s'appuie donc sur le lien familial en cause et choisit une formule qui semble montrer le caractère restrictif des cas pouvant donner lieu à une entrave, rappelant celle utilisée dans la décision Dereci (ce rapprochement est fait par Stephen Coutts).

Dans ses conclusions, l'avocat général propose un raisonnement qui diffère de celui suivi par la Cour sur différents points. Tout d'abord, elle ne considère pas la circulation du 
«travailleur » uniquement au sens de l'article 45 , mais celle du citoyen qui «exerce des droits de libre circulation en liaison avec son emploi» (situation qu'elle rapproche, d'une certaine façon de la liberté "passive» de recevoir des services dans un autre État membre sans s'y rendre - au sens semble-t-il de «sans y séjourner », V. point 124s.). Elle explique que le droit de séjour du membre de la famille doit être apprécié en fonction de trois critères, "le lien familial ", "l'exercice par le citoyen de l'Union de droits de libre circulation » et « le lien de causalité entre la résidence du ressortissant d'un pays tiers et l'exercice par le citoyen de l'Union des droits de libre circulation " (point 121).

41 Par ailleurs, il faut préciser que la Cour ne mentionne pas, dans cet arrêt, que le séjour s'exerce dans les conditions prévues par la directive 2004/38. Le fonctionnement de ce droit de séjour, et les membres de la famille concernés, ne sont donc pas les mêmes que ceux de l'arrêt précédant. Mais elle fournit des éléments d'appréciation qu'il appartient à la juridiction de renvoi de prendre en compte lors de l'examen de la situation. Ainsi, le rôle de la personne au sein de la famille ou l'étroitesse du lien familial peuvent constituer des éléments opportuns pour cette appréciation. En revanche, apparaît indifférent l'État d'établissement de l'employeur (points 38 et 39),En fin de compte, c'est le critère de « nécessité » de celui-ci au regard de l'effectivité du droit de circulation du travailleur qui semble l'unique critère permettant de déterminer les situations pouvant donner lieu à un droit de séjour dérivé.

En définitive, la Cour transpose avec prudence la solution développée pour le prestataire de service au travailleur. De même que dans l'arrêt précédent, ceci a pour conséquence de limiter les "situations purement internes" et cela permet, dans certains cas, la reconnaissance d'un droit de séjour aux ressortissants de pays tiers, membres de la famille d'un citoyen de l'Union dans sont État de nationalité. Dans cet arrêt, les droits découlent de la protection de l'effet utile des libertés de circulation du citoyen, en tant que travailleur et non plus du citoyen en tant que tel comme le premier arrêt. Le caractère "nécessaire » du maintien du membre de la famille pour assurer la liberté de circulation semble difficile à établir. La circulation des solutions et des références entre les différents statuts retient alors l'attention. Il est intéressant de noter que la «transposition» d'une solution définie dans le cas du prestataire de service à celui du travailleur est notamment limitée par des références directes ou indirectes à la jurisprudence récente de la Cour développée dans le champ de la «citoyenneté » pour bien limiter les questions qui relèvent du champ de sa liberté de circulation d'autres lignes jurisprudentielles.

Relevons en conclusion que si la Cour parvient à établir ces solutions, elle ne manque pas de souligner les conditions dans lesquelles un droit de séjour peut être octroyé, et ce sans se référer au droit au respect de la vie privée et familiale en tant que tel. La protection de la famille du citoyen ou du travailleur n'intervient ici qu'en ce qu'elle est au service de leurs libertés de circulation. C'est l' " effet utile » de la liberté de circulation qui permet de fonder en droit de l'Union des obligations pour les États qui touchent à des sujets aussi sensibles que leur politique migratoire à l'égard des membres de la famille de leurs propres ressortissants résidant sur leur territoire. Si la solution dégagée se veut donc protectrice, le raisonnement traduit une certaine prudence de la Cour, manifestement 
sensible aux inquiétudes étatiques. Celle-ci se traduit notamment par l'absence de référence à la "vocation fondamentale" du statut de citoyen ou aux droits fondamentaux, contrairement aux arrêts dont la Cour «transpose» la solution. La protection de ce type de familles peut toutefois être perçue comme participant à la construction progressive du statut du citoyen européen.

CJUE, 12 mars 2014, O. contre Minister voor Immigratie, Integratie en Asiel, et Minister voor Immigratie, Integratie en Asiel contre B., aff.C-456/12. voor Immigratie, Integratie en Asiel contre G., aff.C-457/12.

Les Lettres « Actualités Droits-Libertés » (ADL) du CREDOF (pour s'y abonner) sont accessibles sur le site de la Revue des Droits de l'Homme (RevDH) - Contact

\section{RÉSUMÉS}

La Cour de justice de l'Union européenne a rendu le 12 mars deux arrêts importants sur le droit de séjour des ressortissants de pays tiers, membres de la famille d'un citoyen européen, dans l'État membre d'origine dudit citoyen. En ayant recours à des principes classiques de sa jurisprudence, elle transpose des solutions développées dans d'autres contextes pour accorder, dans certaines situations, des droits de séjours dérivés en vertu de l'" effet utile» des libertés de circulation garanties par les articles 21 et 45 du traité sur le fonctionnement de l'Union européenne (TFUE). Cet ancrage constitue à la fois le fondement et la limite de ces droits de séjour.

\section{AUTEURS}

\section{MARIA GKEGKA}

Doctorante en droit public (Université de Paris Ouest Nanterre La Défense, CREDOF et Université de Paris VIII, Forces du droit)

\section{VINCENT RÉVEILLÈRE}

Doctorant en droit public (Institut Universitaire Européen, Florence) 